Carolien Bonroy*, Sofie Schouwers, Mario Berth, Muriel Stubbe, Yves Piette, Ilse Hoffman, Katrien Devreese and Lieve Van Hoovels

\title{
The importance of detecting anti-DFS70 in routine clinical practice: comparison of different care settings
}

https://doi.org/10.1515/cclm-2017-0541

Received June 21, 2017; accepted January 3, 2018; previously published online February 10, 2018

\section{Abstract}

Background: Screening for antinuclear antibodies by indirect immunofluorescence (ANA-IIF) is essential in the diagnostic workup of ANA-associated autoimmune rheumatic diseases (AARDs). However, also healthy individuals may test positive, making the interpretation challenging. Recent reports suggest that dense fine speckled 70 antibodies (anti-DFS70) may facilitate this challenge. Here, we investigate their clinical importance based on data from four Belgian laboratories (one primary, two secondary and one tertiary care).

Methods: At least one specific DFS70 assay (DFS70 IgG ELISA or lineblot [Euroimmun, full length antigen] and/ or DFS70 IgG CLIA [Inova Diagnostics, truncated antigen]) was performed on four consecutive cohorts of homogeneous-like ANA-IIF samples $(n=697)$. Co-occurrence with AARD-specific ANA and clinical information were documented in the anti-DFS70-positive samples.

Results: Using a combination of solid phase techniques, we found between $7.6 \%$ and $26 \%$ anti-DFS70 in the

\footnotetext{
*Corresponding author: Carolien Bonroy, Department of Laboratory Medicine, Ghent University Hospital, Ghent, Belgium,

Phone: +0032-9-3323631, Fax: +0032-9-3324985,

E-mail: carolien.bonroy@uzgent.be

Sofie Schouwers: Department of Laboratory Medicine, GZA Hospitals, Antwerp, Belgium

Mario Berth: Immunology Department, Algemeen Medisch Laboratorium (AML), Antwerp, Belgium

Muriel Stubbe: Department of Rheumatology, OLV Hospital, Aalst, Belgium

Yves Piette: Department of Rheumatology, Ghent University Hospital, Ghent, Belgium

Ilse Hoffman: Department of Rheumatology, GZA Hospitals, Antwerp, Belgium

Katrien Devreese: Department of Laboratory Medicine, Ghent University Hospital, Ghent, Belgium

Lieve Van Hoovels: Department of Laboratory Medicine, OLV Hospital, Aalst, Belgium
}

different cohorts. Focusing on anti-DFS70 CLIA-positive samples without co-occurrence of AARD-specific ANA, we observed a trend towards lower frequency in tertiary $(8 \%$ $[\mathrm{p}=0.0786])$ and secondary care $(12 \%[\mathrm{p}=0.1275]$ and $6 \%$ [p $<0.001])$ compared to primary care $(21 \%)$. Moreover, in this specific subpopulation, AARD was less frequent (0\%-50\% compared to $6 \%-77 \%$ in the total anti-DFS70positive group).

Conclusions: Anti-DFS70 prevalence depends on the applied assay and care setting. Our data suggest that, for an ANA-IIF-positive patient, it is rather the absence of AARD-associated ANA and clinical symptoms that contribute to the exclusion of AARD than the presence of antiDFS70. Nevertheless, isolated anti-DFS70 helps to clarify positive ANA-IIF results, especially if pretest probability for AARD is low.

Keywords: anti-DFS70/LEDGF; antinuclear antibodies; systemic rheumatic autoimmune diseases.

\section{Introduction}

Antinuclear antibody (ANA) detection is considered essential in the diagnostic workup of ANA-associated autoimmune rheumatic diseases (AARDs) $[1,2]$. In the routine laboratory, indirect immunofluorescence (IIF) on HEp-2(000) cells is the most widely applied screening method [3]. However, because screening for ANA by indirect immunofluorescence (ANA-IIF) lacks specificity with up to $20 \%$ positivity found in apparently healthy individuals, further testing to identify the specific antigenic targets of ANA is essential to improve the diagnostic contribution of the positive ANA-IIF test result [4].

Clinicians may have different reasons for requesting ANA tests. ANA results can be used to support or to assist the clinical diagnosis in patients with symptoms of AARD. In addition, in some AARD (e.g. systemic sclerosis), the presence of specific ANA is also associated with particular disease manifestations emphasizing their use as prognostic markers [5]. However, in daily clinical practice, an evolution in the request pattern is observed with ANA tests 
more frequently ordered in a wider clinical framework. In this context with low pretest probability, a negative ANA-IIF test may contribute to exclude some AARDs like systemic lupus erythematosus, mixed connective tissue disease and systemic sclerosis [4]. Nevertheless, this approach also results in a higher frequency of 'clinically out of context' ANA-IIF positivity, leading to unnecessary follow-up testing, possible misdiagnosis and even incorrect therapies [1]. Recent observations suggest that isolated DFS70 antibodies (anti-DFS70) might contribute to explaining a significant proportion of these AARD-unrelated ANA-IIF-positive results [6].

Anti-DFS70 target the lens epithelium-derived growth factor (LEDGF) and react with a conserved and conformational epitope, restricted to a single region located within the integrase binding domain at the C-terminal of the protein [7]. On ANA-IIF, anti-DFS70 are characterized by a dense fine speckled (DFS) staining of the nucleoplasm of the interphase cells, typically excluding the nucleoli, and by a strong granular-speckled staining of the condensed mitotic chromosomes (for reference images, see website of ICAP [International consensus on Antinuclear Antibody Pattern, http://www.anapatterns.org], pattern AC-2) $[8,9]$.

Anti-DFS70/LEDGF were first described in 1994 in interstitial cystitis and later on in a variety of other nonautoimmune conditions (e.g. atopic dermatitis, asthma, gynecologic syndromes and neoplasia), autoimmune conditions (e.g. autoimmune thyroiditis and AARD such as Sjögren's syndrome and systemic lupus erythematosus) and even healthy donors [7, 10-16].

Several studies observed higher frequencies of antiDFS70 in healthy individuals compared to AARD $[2,12,15$, $17,18]$. In a recent meta-analysis of five studies, a mean prevalence of $7.8 \% \pm 6.2 \%$ (median $7.6 \%$ ) anti-DFS70 in healthy controls was calculated, regardless the detection method used. In the same study, the calculated mean prevalence of anti-DFS70 in AARD was $4.5 \pm 2.8 \%$ (median 3.9\%), with isolated anti-DFS70 reactivity being extremely rare $(0.7 \pm 0.9 \%$ [median $0.45 \%$ ] $)$ [7]. Based on these findings, some authors suggested that isolated anti-DFS70 positivity could be used to exclude the diagnosis of AARD $[2,7]$. By contrast, for other authors, this proposal is difficult to support as the ANA test is usually not requested in healthy individuals, resulting in a substantial part of the anti-DFS70-positive tests in patients with AARD [19]. Their idea was also supported by the limited data available on unselected sera for which routine ANA screening was requested, showing low prevalence of confirmed antiDFS70 (1.7\% $\pm 0.1 \%$ [median 1.7\%]), approaching the situation observed in AARD $[2,7]$.
Various other factors contribute to the controversy on the true clinical significance of anti-DFS70. First, differences in frequencies of anti-DFS70 are observed depending on the detection technique (ANA-IIF, enzyme-linked sorbent immunoassay [ELISA], chemiluminescence immunoassay [CLIA] or immunoblot) used. For instance, Bizzaro and coworkers demonstrated that only $14 \%$ of their initial DFS-ANA-IIF-positive sera, were positive on the DFS70 ELISA. In addition, they also observed important HEp-2 cell line variability [19]. Second, differences between the solid phase assays were also described, probably related to the differences in antigens used [20]. Third, it is to be expected that the true added value of anti-DFS70 detection in a routine context is also dependent on the request pattern and the setting of the laboratory (primary care versus secondary or tertiary care). Based on the idea that anti-DFS70 are more frequent in healthy and that isolated anti-DFS70 are rare in AARD, it is to be expected that in populations with higher pretest probability of AARD, the prevalence of anti-DFS70 is lower and the co-occurrence of anti-DFS70 with other AARD-associated ANA is higher, resulting in lower clinical added value. However, no formal studies have been performed to document this assumption.

The purpose of this multicenter study was to evaluate the added value of detecting anti-DFS70 in different routine contexts. Therefore, we pooled data from four Belgian laboratories in different care settings and compared the frequency and co-occurrence of anti-DFS70 with other AARD-associated ANA. Also demographics and clinical data were collected.

\section{Materials and methods}

\section{Samples}

Consecutive samples of adult patients ( $\geq 18$ years) for which ANA-IIF was requested were included in this study. Samples originated from four Belgian clinical routine laboratories in different care settings: Algemeen Medisch Laboratorium Antwerp (AML) (primary care), OLV Hospital Aalst (OLVA) (secondary care), GZA Hospitals Antwerp (GZA) (secondary care) and University Hospital Ghent (UZG) (tertiary care). The number of samples included and the collection timeframe in each center are shown in Table 1. As the recognition of the DFS70ANA-IIF pattern is difficult, definite inclusion for further anti-DFS70 analysis was based on the presence of a homogeneous ANA IIF pattern (i.e. homogeneous and regular fluorescence of the interphase nuclei and metaphase chromatin) or a speckled mitosis-positive ANA IIF pattern (i.e. all types of speckled fluorescence of interphase nuclei in combination with a speckled aspect of the metaphase chromatin), 


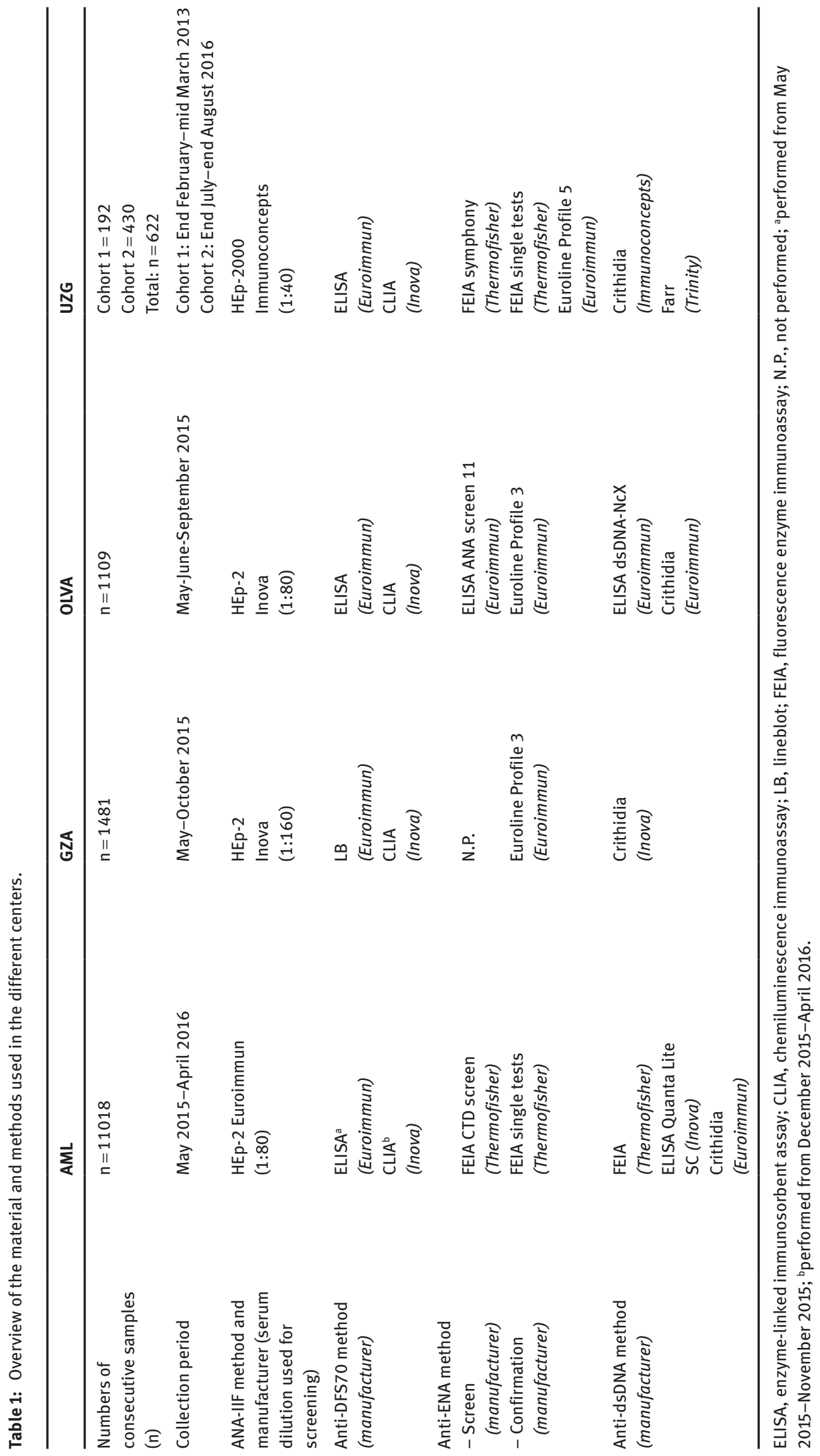


further referred to as the homogeneous-like group. All serum samples were obtained as part of routine screening for ANA. No informed consent was needed for this retrospective study, but the study was performed according to the Declaration of Helsinki and approved by the Local Hospital Ethics Committee.

\section{Routine ANA-IIF analysis and confirmatory tests}

All samples were tested for ANA-IIF, double-stranded DNA antibodies (anti-dsDNA) and extractable nuclear antibodies (anti-ENA) using the routine method in each center. See Table 1 for more details on the methods used. For ANA-IIF analysis, three centers used HEp-2, and one center used HEp-2000. For the detection of anti-ENA, three centers used a screening method with pooled antigens before further identification of the specific reactivities. As the screening dilution for ANA-IIF routine analysis differed between centers, the data were aligned towards a cutoff for positivity of 1:160.

\section{Detection of DFS70 antibodies}

Three different techniques for the detection of anti-DFS70 were used in this study: one enzyme-linked immunosorbent assay (AntiDFS70 ELISA, EUROIMMUN AG, Lübeck, Germany) (DFS70 ELISA), one lineblot (Euroline ANA Profile 3 plus DFS70, EUROIMMUN AG, Lübeck, Germany) (DFS70 LB) and one chemiluminescence immunoassay (QUANTA Flash ${ }^{\circledR}$ DFS70, Inova diagnostics, San Diego, CA, USA) (DFS70 CLIA). The DFS70 ELISA and LB methods use the fulllength (FL) protein (aa 1-530), and the DFS70 CLIA method uses a truncated (TC) antigen (aa 349-435). For all assays, the manufacturers' cutoff was applied. In three centers (GZA, OLVA and UZG) DFS70 antibodies were detected using two different solid phase techniques (see Table 1). In the primary care center (AML), only one technique was used (DFS70 ELISA or CLIA depending on the timeframe). All DFS70-positive samples (positivity with at least one technique) from the secondary and tertiary care centers were also analyzed with an immunoabsorption method (NOVA Lite ${ }^{\circledR}$ HEp-2 Select, Inova diagnostics, San Diego, CA, USA).

\section{Data analysis}

All data evaluations were performed using MedCalc ${ }^{\circledR}$ Software version 15.6.1 (Ostend, Belgium). The Mann-Whitney test was used to evaluate the difference between two independent samples. For comparison of proportions, $\chi^{2}$-testing with Yates' correction for continuity was applied. A statistical significance level of 0.05 was used. In case of multiple testing, the Bonferroni correction was applied.

\section{Results}

\section{Description of the referral pattern}

The demographics of each population and characteristics of the referral pattern per center are given in Table 2. Higher numbers of referrals from rheumatologists were observed in secondary and tertiary care compared to primary care.

\section{ANA-IIF positivity and frequency of the homogeneous-like pattern in the different cohorts}

The highest ANA-IIF positivity in routine was observed in the UZG (58\%) and the OLVA (52\%), whereas lower frequencies were observed in AML (23\%) and GZA (18\%). However, it must be mentioned that UZG applies a lower screening serum dilution (1:40) compared to OLVA (1:80), AML (1:80) and GZA (1:160). At identical cutoff of 1:160, a total of 694 samples showed a homogeneous-like pattern on ANA-IIF. The frequency of this pattern within each individual consecutive cohort was 3\% $(n=327 / 11018)$ for AML, $8 \%(n=50 / 622)$ for UZG, 9\% $(n=138 / 1481)$ for GZA and $19 \%$ for OLVA $(n=211 / 1109)$.

\section{Differences between solid phase assays for anti-DFS70 detection}

In three centers (GZA, OLVA and UZG, $\mathrm{n}=367$ ), DFS70 antibodies were detected using two different solid phase techniques (see Table 1): one technique using the TC antigen (DFS70 CLIA) and one technique using the FL antigen (DFS70 ELISA or LB). Overall, a kappa agreement of 0.749 (95\% confidence interval [CI] 0.659-0.859) between the FL and TC antigen-based assays was observed. In total, $49(13 \%)$ of 367 samples showed reactivity in at least one assay, $63 \%(n=31 / 49)$ of them being positive in both assays. Most single positive samples $(n=17 / 18)$ showed reactivity in an FL antigen assay (DFS70 ELISA or LB).

Table 2: Description of the consecutive sample cohorts and characteristics of the referral pattern per center.

\begin{tabular}{|c|c|c|c|c|}
\hline & $\operatorname{AML}(n=11018)$ & GZA $(n=1481)$ & OLVA $(n=1109)$ & UZG $(n=622)$ \\
\hline Median age (range) & 51 year $(18-101)$ & 54 year $(18-95)$ & 53 year $(18-95)$ & 50 year $(18-89)$ \\
\hline Male/female (female\%) & $3479 / 7539(68.4 \%)$ & $530 / 951(64.2 \%)$ & $344 / 765(69.0 \%)$ & $195 / 427(68.6 \%)$ \\
\hline$\%$ Requests from rheumatologists & $5 \%$ & $26 \%$ & $55 \%$ & $31 \%$ \\
\hline
\end{tabular}




\section{Frequency of anti-DFS70 in the different cohorts}

In total, 134 of 694 homogeneous-like samples were found positive for anti-DFS70 using a combination of the solid phase techniques. See Table 3 for an overview of the positive samples in each center in relation to the different assays. When only anti-DFS70 reactivity on CLIA (55 samples in total) was taken into account to calculate the frequencies over the different cohorts, the highest frequency of anti-DFS70 within the homogeneous-like population was observed in AML $(23 \%, n=23 / 101)$, with a trend towards lower frequency in tertiary care (UZG, $10 \%$, $\mathrm{n}=5 / 50$ ) and secondary care (GZA [13\%, $\mathrm{n}=14 / 106]$, OLVA $[6 \%, n=13 / 211])$. Nevertheless, significance could only be shown for OLVA $(\mathrm{p}<0.001)$.

\section{Co-occurrence of anti-DFS70 with other ANA}

Co-occurrence of anti-DFS70 with other AARD-associated ANA as determined by the routine methods was observed in $9 \%(\mathrm{n}=5)$ of the 55 DFS70 CLIA-positive samples (see Table 3 for individual numbers of the centers). Higher frequencies of co-occurrence were found in the FL assay (DFS70 LB or ELISA) positive samples (31\%, $\mathrm{n}=15 / 48$ [only tertiary and secondary care taken into account]), with up to $71 \%(n=12 / 17)$ co-occurrence in the positive samples without concurrent CLIA reactivity (single FL assay positivity, $\mathrm{p}<0.0001$ ).

The frequency of DFS70 CLIA reactivity without concomitant routinely detected AARD-associated ANA ranged between $6 \%$ and $21 \%$, with a trend towards lower frequency in tertiary care (UZG 8\%, n=4/50) and secondary care (GZA [12\%, $\mathrm{n}=13 / 106]$, OLVA $[6 \%, n=12 / 211]$ ) compared to primary care (AML 21\%, $n=21 / 101)$. However, only the comparison between OLVA and AML was found to be statistically significant $(p<0.001)$

All DFS70-positive samples originating from the secondary and tertiary care centers were also analyzed with the NOVA Lite ${ }^{\circledR}$ HEp-2 Select immunoabsorption method to determine isolated anti-DFS70 reactivity on IIF. Using this approach, $78 \%(n=25 / 32)$ of the DFS70 CLIA positive samples showed a pattern suggestive for isolated antiDFS70 IIF reactivity. By contrast, all samples with single FL assay reactivity $(n=17)$ had an ANA-IIF pattern on HEp-2 Select suggestive of the co-occurrence of other ANA reactivities, and in $64 \%(n=11)$ of them, the specific reactivity was also identified in the routine anti-ENA/dsDNA methods. The most frequently observed reactivity was anti-Scl70 (in 7/17 [41\%]). Other reactivities were anti-Sm $(\mathrm{n}=2)$, anti-Ro52 $(\mathrm{n}=4)$, anti-Ro60 $(\mathrm{n}=3)$, anti-SSB $(\mathrm{n}=1)$ and anti-dsDNA $(\mathrm{n}=1)$.

Table 3: Numbers of samples showing the homogeneous-like pattern and DFS70 reactivity over the different cohorts and in relation to the different solid phase techniques.

\begin{tabular}{|c|c|c|c|c|c|}
\hline Number of samples & Total & AML & GZA & OLVA & UZG \\
\hline Homogeneous-like samples & 694 & $327^{a}$ & $106^{\mathrm{b}}$ & 211 & 50 \\
\hline $\begin{array}{l}\text { Anti-DFS70-positive samples based on positivity with } \\
\text { at least } 1 \text { technique [\% of homogeneous-like samples] }\end{array}$ & $134[19 \%]$ & $85^{\mathrm{a}}[26 \%]$ & 20 [19\%] & $16[7.6 \%]$ & $13[26 \%]$ \\
\hline \multicolumn{6}{|l|}{$\begin{array}{l}\text { Anti-DFS70-positive samples in relation to the applied } \\
\text { technique }\end{array}$} \\
\hline$-\mathrm{CLIA}+$ & 55 & 23 & $14^{\mathrm{c}}$ & $13^{c}$ & $5^{c}$ \\
\hline - FL assay+ & 110 & 62 & 19 & 16 & 13 \\
\hline - Single CLIA+ & $1^{\mathrm{e}}$ & $N A^{d}$ & 1 & 0 & 0 \\
\hline - Single FL assay+ & $17^{\mathrm{e}}$ & $N A^{d}$ & 6 & 3 & 8 \\
\hline \multicolumn{6}{|l|}{$\begin{array}{l}\text { Anti-DFS70-positive samples without concurrent ANA- } \\
\text { reactivity in relation to the applied technique }\end{array}$} \\
\hline$-\mathrm{CLIA}+$ & 50 & 21 & 13 & 12 & 4 \\
\hline - FL assay+ & 91 & 58 & 15 & 13 & 5 \\
\hline - Single CLIA+ & $1^{\mathrm{e}}$ & NA & 1 & 0 & 0 \\
\hline - Single FL assay+ & $5^{e}$ & NA & 3 & 1 & 1 \\
\hline
\end{tabular}

CLIA, chemiluminescence immunoassay using the truncated antigen; FL assay, lineblot or enzyme-linked sorbent assay using the full-length antigen; NA, not applicable. ${ }^{2}$ One hundred and one samples were analyzed with CLIA of which 23 samples were positive on DFS70 CLIA;

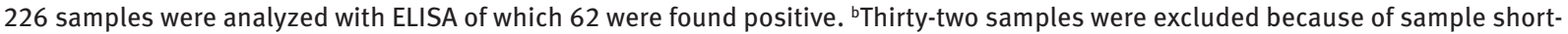
age or double inclusion in the consecutive series. 'All (except 1 GZA sample) showed double reactivity for CLIA and a full-length antigenbased assay (lineblot or ELISA). ${ }^{\mathrm{S}}$ Samples were analyzed by either CLIA or ELISA. e Only data from secondary and tertiary care centers were included. 'Definition of concurrent ANA reactivity based on the routine anti-ENA/dsDNA tests. 


\section{Demographic and clinical characterization of the anti-DFS70-positive samples}

We evaluated the characteristics of the isolated antiDFS70 CLIA-positive samples (comparison of isolated DFS70+ versus isolated DFS70-). The results are summarized in Table 4. A female dominance in the isolated anti-DFS70-positive cohorts was observed for three out of the four centers without significant difference. For two centers, the isolated anti-DFS70-positive patient group was significantly younger than the negative group.
No significant differences in the proportion of ANA-IIF titers $\geq 1 / 160$ were observed.

We also documented the association with AARD in the anti-DFS70-positive samples of three cohorts (UZG, GZA and OLVA) (Table 5) by retrospective review of the medical records and laboratory data. Considering all DFS70-positive samples (at least one method DFS70 positive), the highest association between AARD and antiDFS70 positivity was observed in UZG (77\%, $\mathrm{n}=10 / 13)$. A lower frequency of AARD was observed in the secondary care centers $(25 \%[n=5 / 20, \mathrm{GZA}]$ and $6 \%[n=1 / 16$, OLVA $])$.

Table 4: Characteristics of the isolated anti-DFS70 CLIA-positive samples.

\begin{tabular}{|c|c|c|c|c|}
\hline Number of samples & AML & GZA & OLVA & UZG \\
\hline $\begin{array}{l}\text { Number of isolated anti-DFS70 CLIA+ } \\
\text { samples within the homogeneous-like cohort } \\
\text { Female, \% }\end{array}$ & $21 / 101^{\mathrm{a}, \mathrm{b}}$ & $13 / 106^{\mathrm{a}}$ & $12 / 211^{\mathrm{a}}$ & $4 / 50^{a}$ \\
\hline Isolated DFS70+ & $76 \%$ & $82 \%$ & $80 \%$ & $100 \%$ \\
\hline Isolated DFS70- & $89 \%$ & $76 \%$ & $75 \%$ & $83 \%$ \\
\hline p-Value $\left(\chi^{2}\right)$ & $p=0.2595$ & $\mathrm{p}=0.9373$ & $p=0.9923$ & $\mathrm{p}=0.8422$ \\
\hline \multicolumn{5}{|l|}{ Age (median, [range]) } \\
\hline Isolated DFS70+ & $41[25-83]$ & $41[21-82]$ & $46[20-76]$ & $51[28-56]$ \\
\hline Isolated DFS70- & 59 [19-91] & $57[22-88]$ & 57 [24-91] & $51[19-86]$ \\
\hline p-Value (Mann-Whitney)c & $p=0.00005$ & $p=0.2612$ & $p=0.0253$ & $p=0.6041$ \\
\hline \multicolumn{5}{|l|}{ ANA-IIF titer ( $\%$ with titer $\leq 1 / 160)$} \\
\hline Isolated DFS70+ & $81 \%$ & $54 \%$ & $25 \%$ & NP \\
\hline Isolated DFS70- & $68 \%$ & $37 \%$ & $36 \%$ & NP \\
\hline $\mathrm{p}$-Value $\left(\chi^{2}\right)$ & $p=0.0924$ & $p=0.2404$ & $p=0.4469$ & \\
\hline
\end{tabular}

CLIA, chemiluminescence immunoassay; NP, not performed. a Definition of isolated reactivity based on the anti-dsDNA and anti-ENA routine assays. ${ }^{b}$ For AML, only samples performed on CLIA $(n=101)$ were taken into account. 'Significant difference shown in bold.

Table 5: SARD association in anti-DFS70-positive samples.

\begin{tabular}{|c|c|c|c|}
\hline & GZA & OLVZ & UZG \\
\hline \multicolumn{4}{|c|}{$\begin{array}{l}\text { Anti-DFS70-positive samples } \\
\text { (positivity with at least one technique) }\end{array}$} \\
\hline Number & 20 & 16 & 13 \\
\hline n SARD+ & 5 & 1 & 10 \\
\hline$\%$ SARD+ & $25 \%$ & $6 \%$ & $77 \%$ \\
\hline Type SARDa & $\operatorname{SLE}(n=5)$ & $\operatorname{SSc}(n=1)$ & $\operatorname{SLE}(n=1), \operatorname{SSc}(n=9)$ \\
\hline \multicolumn{4}{|c|}{ Anti-DFS70 CLIA+ } \\
\hline Number & 14 & 13 & 5 \\
\hline n SARD+ & $1 / 13^{b}$ & $0 / 13$ & $3 / 5$ \\
\hline$\%$ SARD+ & $8 \%$ & $0 \%$ & $60 \%$ \\
\hline Type SARDa & $\operatorname{SLE}(n=1)$ & & $\operatorname{SLE}(n=1), \operatorname{SSc}(n=2)$ \\
\hline \multicolumn{4}{|c|}{ Isolated anti-DFS70 CLIA $+^{\mathrm{b}}$} \\
\hline Number & 11 & 10 & 4 \\
\hline n SARD+ & $0 / 10^{c}$ & $0 / 10$ & $2 / 4$ \\
\hline$\%$ SARD + & $0 \%$ & $0 \%$ & $50 \%$ SARD \\
\hline Type SARDa & & & SLE (1), SSc (1) \\
\hline
\end{tabular}

SLE, systemic lupus erythematosus; SSc, systemic sclerosis. ${ }^{a}$ Association with SARD documented based on retrospective review of the

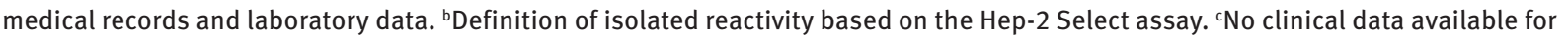
1 anti-DFS70-positive patient. 
Focusing on the isolated anti-DFS70 CLIA-positive samples defined using HEp-2 select analysis, the association with AARD was lower ( $50 \%$ in UZG, $\mathrm{n}=2 / 4$ ) and absent in the secondary care centers.

\section{Strategy for routine analysis}

We also evaluated the possible routine impact of detecting DFS70 antibodies on every homogeneous-like sample without concomitant AARD-associated ANA. We calculated that extra anti-DFS70 testing would be necessary in $16 \%$ of the 1109 consecutive samples in OLVA ( 173 samples showed a homogeneous-like pattern on IIF with no AARD-associated ANA in the routine tests), in $5 \%$ for UZG $(\mathrm{n}=30 / 622)$, in $4 \%$ for GZA $(\mathrm{n}=62 / 1481)$ and in $2 \%$ for AML ( $\mathrm{n}=91 / 4180$, consecutive samples analyzed from December 2015 to April 2016). The chances of finding antiDFS70 in these samples eligible for testing were $21 \%$ (GZA, $\mathrm{n}=13 / 62), 13 \%$ (UZG, $\mathrm{n}=4 / 30), 7 \%$ (OLVZA, $\mathrm{n}=12 / 173)$ and $23 \%(n=21 / 91)$ for AML. The frequencies of isolated antiDFS70 within the homogeneous-like cohorts (UZG 8\%, GZA $12 \%$, OLVA 6\% and AML 21\% - see above) match the estimated decrease in anti-ENA/dsDNA testing based on the assumption that no testing will be performed during follow-up in case of isolated anti-DFS70 positivity.

\section{Discussion}

Today, it is accepted that screening for ANA by IIF remains indispensable in the differential diagnosis of AARD [3]. In addition, ANA-IIF analysis is, more frequently than before, ordered in a wider clinical framework, resulting in lower pretest probability of AARD. Against this background of a less focused request pattern, several authors reported the presence of anti-DFS70 antibodies (1\%-15\%) in patients referred for ANA testing [2, 21-26]. In our study on different consecutive populations referred for ANA testing, we detected $0.5 \%$ anti-DFS-70 using CLIA in primary care, $0.9 \%-1.2 \%$ in secondary care and $0.9 \%$ in tertiary care. Therefore, we cannot confirm the suggestion of Seelig and colleagues that a lower prevalence of DFS-70 antibodies is to be expected with higher quota of AARD patients within the clientele [7]. Nevertheless, we observed a trend towards lower frequency of anti-DFS70 within the homogeneous-like samples on ANA-IIF in tertiary care (10\%) and secondary care (13\% and $6 \%$ ) compared to primary care $(23 \%)$. Previous findings showing that anti-DFS70 were more frequent in younger patients were confirmed in three out of four cohorts. By contrast, significance could not be shown for the previously reported higher frequency of females [15, 17].

The laboratory identification of anti-DFS70 has some important challenges. Indeed, differences in frequency of anti-DFS70 are observed depending on the detection technique used, and even within methods, variability is observed [17, 19, 20]. For ANA-IIF, the differences have been assigned to the subjectivity of the interpretation as well as to substrate variability [17, 19]. Moreover, some authors also suggested that in a routine context, the frequent mixed homogeneous and speckled patterns or quasi-homogeneous patterns are difficult to distinguish from the characteristic DFS70 ANA-IIF pattern [27]. In addition, it has been postulated that there are likely also other antigen targets that produce a DFS IIF pattern. One target recently suggested was MeCP2 (methyl CpG protein 2 ), but the presence of antibodies targeting this antigen could not be confirmed in human sera [28]. This variability/difficulty was also observed in our study with differences observed between HEp-2 and HEp-2000 results as well as interreader variability (data not shown). To overcome this, we did not use the criterion of the characteristic DFS pattern for inclusion for further testing, but we applied the broader homogeneous-like criterion. The frequency of the homogeneous-like pattern within each cohort ranged between 3\% and 19\% (at titer 1:160). The higher frequency of homogeneous-like samples in one of the secondary care centers compared to tertiary care is in line with the higher frequency of requests originating from the rheumatology department.

We also compared anti-DFS70 data from different solid phase assays. Our results revealed a substantial agreement between CLIA (based on TC antigen) and the FL antigen assays (DFS70 ELISA or LB, both from the same manufacturer). However, it was remarkable that the discordant samples were mostly single positive with the FL antigen assays. Our observations are in line with the findings of Bizzaro and colleagues [20], who also identified more anti-DFS70 using Euroimmun assays compared to CLIA. Theoretically, this higher sensitivity of the FL antigen assays compared to TC antigen assays, could be related to targeted epitopes not included in the TC recombinant antigen. Nevertheless, their results suggested that the differences in the assays are not reducible to differences in type of antigen used (TC vs. FL), as higher sensitivity compared to CLIA was also observed with another TC antigen assay. The same authors also suggested a possible impact of the applied cutoff for positivity. This was compatible with the results of our study, as most discordant samples showed low positivity in the FL assays (data not shown) and presence 
of AARD-related ANA. In addition, we observed in an unselected consecutive cohort of routine samples (UZG, $n=186)$ that discordant anti-DFS70 samples $(n=8 / 9)$ did not show an isolated homogeneous-like pattern on ANA-IIF (data not shown). Within this context, we suggest not to report anti-DFS70 in absence of an isolated homogeneous-like pattern.

Recent observations suggest that especially antiDFS70 in absence of other AARD-related ANA (referred to as 'isolated anti-DFS70') might contribute to explaining a significant proportion of these AARD-unrelated ANAIIF-positive results $[7,8]$. Within this context, we would also like to highlight that the definition of isolated antiDFS70 differs between studies. We used in our study two approaches: (1) based on negative results in the routine anti-ENA/dsDNA assays and (2) based on the disappearance of the DFS-pattern on IIF after immunoabsorption. The latter approach allows for a more narrow definition of isolated anti-DFS70 as also ANA-IIF-positive reactivities not included in the classical anti-ENA assays are detectable. In our study, co-occurrence of anti-DFS70 with routinely detected AARD-associated ANA was mostly observed in the FL DFS70-positive samples (overall 31\%, up to $71 \%$ in the subset with single FL antigen reactivity). In the samples with CLIA DFS70 reactivity, co-occurrence with routinely detected AARD-associated ANA was lower $(7 \%-20 \%)$, with the highest frequency observed in tertiary care. In these samples, the highest AARD-association was also observed (see also below). Globally, these observations suggest a higher specificity of DFS70 CLIA and highlight the importance of focusing on the isolated presence of anti-DFS70.

Some authors suggested that the isolated presence of anti-DFS70 could be used as an exclusion marker for AARD, a suggestion based on the observation of lower prevalence of anti-DFS70 in AARD (median 3.9\%) compared to healthy persons (7.6\%) [7]. This idea was also supported by the lower median prevalence of isolated anti-DFS70 in AARD patients $(<1 \%)$ [7]. Nevertheless, in healthy donors, the coincidence of anti-DFS70 with ANA, and especially AARD-associated ANA, was not always thoroughly investigated [7]. We found between $0 \%$ and $77 \%$ AARD association within our anti-DFS70-positive cohort (depending on the definition of anti-DFS70 positivity and cohort). Therefore, our data do not support that anti-DFS70 can exclude AARD. To our opinion, in a positive ANA IIF sample, it is rather the absence of a AARDrelated ANA and clinical symptoms that contributes in the exclusion of AARD than the presence of anti-DFS70. Nevertheless, we can agree that anti-DFS70 may explain positive ANA-IIF results, contributing in the clarification of diagnostic challenges.

Some authors claimed that measurements of antiDFS70 are cost-effective in patients with AARD suspicion by comparing costs of follow-up laboratory testing and follow-up clinic visits after integration of anti-DFS70 analysis in their workup algorithm [29]. They calculated a cost saving of laboratory costs of about 17,000 euros by applying their new algorithm. This was based on the presence of anti-DFS70 in 23 samples of a total of 170 ANA-IIF homogeneous or speckled positive samples (13\%). Unfortunately, details on the result calculations are not mentioned, but a decrease of $50 \%$ for anti-ENA and $75 \%$ for anti-dsDNA after implementation of their new approach is described. For comparison, we estimated a decrease of $8 \%-21 \%$ in anti-ENA/dsDNA tests based on the assumption that no testing will be performed during follow-up in case of isolated anti-DFS70 positivity. These differences emphasize on the importance of taking into account the care setting (or alternatively the referral pattern or origin of the requests) when performing cost-effectiveness studies.

Our study has some limitations. First, as this study followed out of the real routine laboratory situation, all centers used their established routine method for ANA-IIF and the detection of anti-ENA and anti-dsDNA. Therefore, we cannot exclude possible differences between centers in the definition of homogeneous-like samples as well as the definition of samples without AARD-associated ANA. Second, our study did not take into account possible differences between the two methods using FL antigen as both methods were produced by the same manufacturer, and only one assay with truncated antigen was performed. Third, the clinical association study was limited to the secondary and tertiary care samples.

In conclusion, the anti-DFS70 prevalence depends on the applied assays and the care setting, with anti-DFS70 CLIA positivity being most prevalent in primary care. Our data suggest that, in a patient with a positive ANA-IIF result, it is rather the absence of a AARD-associated ANA and clinical symptoms that contribute to the exclusion of AARD than the presence of anti-DFS70. Nevertheless, antiDFS70 in absence of AARD-associated ANA may contribute to the clarification of positive ANA-IIF results, especially if pretest probability for AARD is low. Cost-effectiveness studies are useful but should take into account the care setting/referral pattern/origin of the requests.

Acknowledgments: We thank Euroimmun and Werfen/ Inova Diagnostics for the donation of the assays. We are 
grateful to Julie Jacobs and Jelle Smet (OLVA), Astrid Muyldermans (GZA) and the laboratory technicians of the different participating centers for their most valuable efforts. Author contributions: All the authors have accepted responsibility for the entire content of this submitted manuscript and approved submission.

Research funding: None declared.

Employment or leadership: None declared.

Honorarium: None declared.

Competing interests: The funding organization(s) played no role in the study design; in the collection, analysis, and interpretation of data; in the writing of the report; or in the decision to submit the report for publication.

\section{References}

1. Mahler M, Meroni PL, Bossuyt X, Fritzler MJ. Current concepts and future directions for the assessment of autoantibodies to cellular antigens referred to as anti-nuclear antibodies. J Immunol Res 2014;2014:315179.

2. Mahler M, Parker T, Peebles CL, Andrade LE, Swart A, Carbone Y, et al. Anti-DFS70/LEDGF antibodies are more prevalent in healthy individuals compared to patients with systemic autoimmune rheumatic diseases. J Rheumatol 2012;39:2104-10.

3. Agmon-Levin N, Damoiseaux J, Kallenberg C, Sack U, Witte T, Herold $M$, et al. International recommendations for the assessment of autoantibodies to cellular antigens referred to as antinuclear antibodies. Ann Rheum Dis 2014;73:17-23.

4. Op De Beeck K, Vermeersch P, Verschueren P, Westhovens R, Marien G, Blockmans D, et al. Detection of antinuclear antibod ies by indirect immunofluorescence and by solid phase assay. Autoimmun Rev 2011;10:801-8.

5. Bonroy C, Smith V, Van Steendam K, Van Praet J, Deforce D, Devreese $\mathrm{K}$, et al. The integration of the detection of systemic sclerosis-associated antibodies in a routine laboratory setting: comparison of different strategies. Clin Chem Lab Med 2013;51:2151-60.

6. Miyara M, Albesa R, Charuel JL, El Amri M, Fritzler MJ, GhillaniDalbin $P$, et al. Clinical phenotypes of patients with anti-DFS70/ LEDGF antibodies in a routine ANA referral cohort. Clin Dev Immunol 2013;2013:703759.

7. Seelig CA, Bauer O, Seelig HP. Autoantibodies against DFS70/ LEDGF exclusion markers for systemic autoimmune rheumatic diseases (SARD). Clin Lab 2016;62:499-517.

8. Ochs RL, Mahler M, Basu A, Rios-Colon L, Sanchez TW, Andrade LE, et al. The significance of autoantibodies to DFS70/LEDGFp75 in health and disease: integrating basic science with clinical understanding. Clin Exp Med 2016;16:273-93.

9. Chan EK, Damoiseaux J, de Melo Cruvinel W, Carballo OG, Conrad K, Francescantonio PL, et al. Report on the second International Consensus on ANA Pattern (ICAP) workshop in Dresden 2015. Lupus 2016;25:797-804.

10. Ochs RL. Autoantibodies and interstitial cystitis. Clin Lab Med 1997;17:571-9.
11. Yamada K, Senju S, Shinohara T, Nakatsura T, Murata Y, Ishihara $M$, et al. Humoral immune response directed against LEDGF in patients with VKH. Immunol Lett 2001;78:161-8.

12. Ochs RL, Muro Y, Si Y, Ge H, Chan EK, Tan EM. Autoantibodies to DFS $70 \mathrm{kd} /$ transcription coactivator p75 in atopic dermatitis and other conditions. J Allergy Clin Immunol 2000;105(6 Pt 1):1211-20.

13. Muro Y, Sugiura K, Morita Y, Tomita Y. High concomitance of disease marker autoantibodies in anti-DFS70/LEDGF autoantibodypositive patients with autoimmune rheumatic disease. Lupus 2008;17:171-6.

14. Ayaki M, Ohoguro N, Azuma N, Majima Y, Yata K, Ibaraki N, et al. Detection of cytotoxic anti-LEDGF autoantibodies in atopic dermatitis. Autoimmunity 2002;35:319-27.

15. Watanabe A, Kodera M, Sugiura K, Usuda T, Tan EM, Takasaki Y, et al. Anti-DFS70 antibodies in 597 healthy hospital workers. Arthritis Rheum 2004;50:892-900.

16. Ochs RL, Stein TW, Jr., Peebles CL, Gittes RF, Tan EM. Autoantibodies in interstitial cystitis. J Urol 1994;151:587-92.

17. Mariz HA, Sato El, Barbosa SH, Rodrigues SH, Dellavance A, Andrade LE. Pattern on the antinuclear antibody-HEp-2 test is a critical parameter for discriminating antinuclear antibodypositive healthy individuals and patients with autoimmune rheumatic diseases. Arthritis Rheum 2011;63:191-200.

18. Kuwabara N, Itoh Y, Igarshi T, Fukunaga Y. Autoantibodies to lens epithelium-derived growth factor/transcription co-activator P75 (LEDGF/P75) in children with chronic nonspecific complaints and with positive antinuclear antibodies. Autoimmunity 2009;42:492-6.

19. Bizzaro N, Tonutti E, Villalta D. Recognizing the dense fine speckled/lens epithelium-derived growth factor/p75 pattern on HEP-2 cells: not an easy task! Comment on the article by Mariz et al. Arthritis Rheum 2011;63:4036-7; author reply 7-8.

20. Bizzaro N, Pesente F, Cucchiaro F, Infantino M, Tampoia M, Villalta D, et al. Anti-DFS70 antibodies detected by immunoblot methods: A reliable tool to confirm the dense fine speckles ANA pattern. J Immunol Methods 2016;436:50-3.

21. Schmeling H, Mahler M, Levy DM, Moore K, Stevens AM, Wick J, et al. Autoantibodies to dense fine speckles in pediatric diseases and controls. J Rheum 2015;42:2419-26.

22. Dellavance A, Viana VS, Leon EP, Bonfa ES, Andrade LE, Leser PG. The clinical spectrum of antinuclear antibodies associated with the nuclear dense fine speckled immunofluorescence pattern. J Rheum 2005;32:2144-9.

23. Bizzaro N, Tonutti E, Visentini D, Alessio MG, Platzgummer S, Morozzi G, et al. Antibodies to the lens and cornea in antiDFS70-positive subjects. Ann N Y Acad Sci 2007;1107:174-83.

24. Pazini AM, Fleck J, dos Santos RS, Beck ST. Clinical relevance and frequency of cytoplasmic and nuclear dense fine speckled patterns observed in ANA-HEp-2. Revista Brasileira de Reumatologia 2010;50:655-60.

25. Fitch-Rogalsky C, Steber W, Mahler M, Lupton T, Martin L, Barr SG, et al. Clinical and serological features of patients referred through a rheumatology triage system because of positive antinuclear antibodies. PloS one 2014;9:e93812.

26. Shovman O, Gilburd B, Chayat C, Amital H, Langevitz P, Watad $A$, et al. Prevalence of anti-DFS70 antibodies in patients with and without systemic autoimmune rheumatic diseases. Clin Exp Rheumatol 2017. PMID: 28770702 [Epub ahead of print]. 
27. Bentow C, Fritzler MJ, Mummert E, Mahler M. Recognition of the dense fine speckled (DFS) pattern remains challenging: results from an international internet-based survey. Auto Immun Highlights 2016;7:8

28. Basu A, Woods-Burnham L, Ortiz G, Rios-Colon L, Figueroa J, Albesa $R$, et al. Specificity of antinuclear autoantibodies recognizing the dense fine speckled nuclear pattern: preferential tar- geting of DFS70/LEDGFp75 over its interacting partner MeCP2. Clin Immunol 2015;161:241-50.

29. Gundin S, Irure-Ventura J, Asensio E, Ramos D, Mahler M, Martinez-Taboada V, et al. Measurement of anti-DFS70 antibodies in patients with ANA-associated autoimmune rheumatic diseases suspicion is cost-effective. Auto Immun Highlights 2016;7:10 